OPEN ACCESS

Edited by:

Gabriel Sandblom,

Karolinska Institute (KI), Sweden

Reviewed by:

John Griniatsos,

Laiko General Hospital of Athens,

Greece

Nadia A. Henriksen,

University of Copenhagen, Denmark Atıl Çakmak,

Ankara University, Turkey

*Correspondence:

Rhiannon L. Harries

rhiannon.harries@doctors.org.uk

Specialty section:

This article was submitted to Visceral Surgery,

a section of the journal

Frontiers in Surgery

Received: 05 January 2018

Accepted: 13 March 2018

Published: 04 April 2018

Citation:

Harries RL. and Torkington J (2018) Stomal Closure: Strategies to

Prevent Incisional Hernia.

Front. Surg. 5:28.

doi: $10.3389 /$ fsurg.2018.00028

\section{Stomal Closure: Strategies to Prevent Incisional Hernia}

\author{
Rhiannon L. Harries* and Jared Torkington \\ Department of Colorectal Surgery, University Hospital of Wales, Cardiff, United Kingdom
}

Incisional hernias following ostomy reversal occur frequently. Incisional hernias at the site of a previous stoma closure can cause significant morbidity, impaired quality of life, lead to life-threatening hernia incarceration or strangulation and result in a significant financial burden on health care systems Despite this, the evidence base on the subject is limited. Many recognised risk factors for the development of incisional hernia following ostomy reversal are related to patient factors such as age, malignancy, diabetes, COPD, hypertension and obesity, and are not easily correctable. There is a limited amount of evidence to suggest that prophylactic mesh reinforcement may be of benefit to reduce the post stoma closure incisional hernia rate but a further large scale randomised controlled trial is due to report in the near future. There appears to be weak evidence to suggest that surgeons should favour circular, or "purse-string" closure of the skin following stoma closure in order to reduce the risk of SSI, which in turn may reduce incisional hernia formation. There remains the need for further evidence in relation to suture technique, skin closure techniques, mechanical bowel preparation and oral antibiotic prescription focusing on incisional hernia development as an outcome measure. Within this review, we discuss in detail the evidence base for the risk factors for the development of, and the strategies to prevent ostomy reversal site incisional hernias.

Keywords: ostomy reversal, incisional hernia, stoma site herniation, stoma closure, stoma closure site hernia, lleostomy, colostomy, hernia

\section{INTRODUCTION}

The term "stoma" is derived from the Greek word meaning "mouth" or "opening" and is used to describe the creation of an artificial opening made into a hollow organ, on the surface of the body. A stoma is also sometimes known as an ostomy. The first recorded surgical creation of a stoma was by a French surgeon Pillore in 1776. Within gastrointestinal surgery, stomas are most commonly formed as either an ileostomy or colostomy. These may be permanent or temporary and may be formed from either the end of the bowel or the side of the bowel still in continuity (known as a loop stoma). Temporary stomas, which may be reversed at a later date, are most frequently created in order to divert intestinal contents away from a distal anastomosis or obstructing lesion (known as a defunctioning stoma) or prior to a second operation to restore bowel continuity after surgery for complicated diverticular disease, inflammatory bowel disease or in the case of an obstructing cancer. The most frequent temporary stoma is the defunctioning ileostomy and closure involves the complete freeing of the bowel from all layers of the surrounding abdominal wall, followed by the anastomosis of both the proximal limb and distal limb of bowel to restore bowel continuity. The resultant defect in the abdominal wall is repaired by closure of the musculoaponeurotic layer. Stoma closure can 
be associated with significant morbidity, including anastomotic leak, obstruction, wound dehiscence, wound infection and the development of an incisional hernia (1-4).

Incisional hernias are defined as "abdominal wall defects, with or without a bulge, around postoperative scars, perceptible or palpable by clinical examination or imaging" according to the European Hernia Society (5). Incisional hernias are common following abdominal surgery, and are likely to develop in the early post-operative period due to separation of the aponeurotic edges. Incisional hernias can cause significant morbidity including pain, altered body image, impaired quality of life (6) and patients can potentially suffer from life-threatening hernia incarceration (6-15\%) or strangulation (2\%), for which emergency surgery may be necessary (7). Not only do incisional hernias have a significant effect on the patients who suffer with them, but they also pose a significant financial burden on health care systems (8). Repair of incisional hernias (both elective or emergency) are performed frequently, with a total of 12,433 incisional hernia repairs being performed in England alone during 2015-16 (9). The cost per primary incisional hernia repair has been estimated at roughly $\$ 16,000$ in the United States (10) and 6451€ in France (11).

\section{METHODS}

The following sources were searched, Cochrane Library, Ovid, Embase, and Medline using PubMed, with the search terms "ileostomy", "colostomy", "ostomy", "stoma”, "reversal", "closure”, "hernia", "incisional", "surgical site infection" singly or in combination. We supplemented these sources with hand searchs of selected articles and clinical trials registries to find relevant articles.

\section{INCISIONAL HERNIAS FOLLOWING STOMA CLOSURE: HOW BIG IS THE PROBLEM?}

A meta-analysis published in 2012 investigated the incidence of incisional hernia following closure of stoma, and included 34 studies and a total of 2,729 unique stoma closures (12). Of the stoma closures included, the majority as anticipated were loop ileostomies (47.6\%), with the commonest indication for stoma formation being related to colorectal cancer $(60.8 \%)$. The overall mean incisional hernia rate following stoma closures was $7.4 \%$, with a wide variation across the studies from $0-48 \%$ with a median follow-up duration of 36 months. The authors reported a lower risk of hernia following reversal of ileostomy when compared to colostomy on meta-analysis, 4 vs $10 \%$ respectively (Odds Ratio (OR) 0.28 , $95 \%$ CI $0.12-0.65 ; p=0.003$ ). The reoperation rate for incisional hernia at a previous stoma site was extracted from 12 of the included studies, and was found to be $4 \%$. A further systematic review found a similar incidence for stoma site incisional hernias to be $8.3 \%(0-33.9 \%)$ (13). However, when the authors only included studies with a low risk of bias the incidence was closer to $30 \%$.

Two factors should be noted with regard to the incidence of stoma site hernia. Firstly, that the long-term risk is not known and secondly, that clinical examination alone is shown to have a lower detection rate of incisional hernia post stoma closure when compared to clinical imaging $(14,15)$. Therefore, studies focusing on only clinical examination may be underestimating the prevalence, as radiological detected herniae may become symptomatic over time and may be missed in studies with a short follow-up period.

\section{WHAT ARE THE RISK FACTORS FOR THE DEVELOPMENT OF INCISIONAL HERNIA POST STOMA CLOSURE?}

There is a plethora of evidence describing risk factors for incisional hernia formation, and these can be broadly classified as either patient associated factors or surgical related factors. Patient associated factors include advancing age, male gender, high body mass index (BMI), cachexia, smoking, diabetes mellitus, immunosuppression, glucocorticosteroid use, oral anticoagulants, connective tissue disorders, jaundice, respiratory disorders, and known malignancy (16-28). Many of these patients associated factors may not be correctable prior to surgery. Surgical related factors include emergency surgery, contaminated surgery, abdominal distension, return to theatre, multiple operations, post-operative respiratory failure, suture choice and closure technique $(7,16,29-32)$. Most of these risk factors act by either increasing the risk of surgical-site infections (SSI) and wound dehiscence, or by delaying the normal wound healing process $(33,34)$. Most of the evidence for risk factors comes from work on midline incisions. Studies focusing on incision hernias following stoma closure are few in number, and will be discussed further.

Cingi et al (2008) reported a cohort study of 66 patients who had stoma reversal (35). Patients who had incisional hernia at the laparotomy wound were found to have an increased risk of having an incisional hernia at the stoma closure site (OR 4.4). The study found no difference in the development of incisional hernias at stoma closure site when comparing age, gender, BMI or time to stoma closure. Guzmán-Valdivia (2008) found an increased risk of incisional hernia at stoma site in patients with concomitant disease such as diabetes, COPD and hypertension, $p=0.005$, however numbers in each arm were small (11/22 cf. 12/48 without concomitant disease) (36).

Schreinemacher et al (2011) performed a retrospective cohort study of 150 consecutive patients who underwent stoma reversals over a 4 year period (37). The authors found a hernia prevalence of $32.4 \%$ within the cohort. The only significant risk factor found to be predictive of incisional hernia at stoma closure site was obesity (OR 5.53; 95\% CI 1.72-17.80). Gender, age, ASA status, COPD, underlying disease indication for stoma, peritonitis at time of stoma formation, length to stoma reversal or suture material for musculoaponeurotic layer were not found to be predictive risk factors. A further case-control study found that malignancy and diabetes were independently predictive of incisional hernia at stoma closure site (OR 21.93, 95\% CI 1.58-303.95 and OR 20.98, 95\% CI 3.23-136.31, respectively) (38). 
Liang et al (2013) compared rates of SSI in consecutive patients undergoing stoma reversals over a 6 year period (39). In 138 patients, they found that history of fascial dehiscence (OR 16.9, 95\% CI 1.94-387), colostomy (OR 5.07, 95\% CI 2.12-13.0), thicker subcutaneous fat (OR 2.02; 95\% 1.33-3.21) and black race (OR 0.35 , 95\% CI 0.13-0.86) were independent risk factors on multivariate analysis. Shah et al (2015) found the following as risk factors for incisional hernia following stoma reversal at a median of 30 months' follow-up on retrospective review; age, diabetes, end colostomies, loop colostomies, BMI > 30 and urgent operation (40).

Brook et al (2016) examined 193 loop ileostomy reversals in order to predict risk factors for hernia development post stoma reversal (41). The results demonstrated that patients experiencing ileostomy site hernia were more likely to have a higher BMI, higher blood pressure at preoperative assessment, colorectal cancer indication for ileostomy formation or postoperative complication occurrence. Logistic regression estimated that for every one unit increase in BMI there was an increase in the OR of 1.2 times the risk of developing a hernia at the stoma closure site. Significance of hypertension persisted on multiple regression independent of ASA status and BMI (OR 18.3), suggesting an intrinsic association of hypertension and stoma closure site hernia development, which the authors suggest could be due to inappropriate activation of inflammatory cytokines causing disordered wound healing (42). The time to ileostomy reversal, initial operative approach (laparoscopic versus open), initial operative urgency (emergency versus elective), consultant surgeon presence, preoperative stoma marking, patient age, gender, smoking status, chemotherapy and suture material choice for musculoaponeurotic layer were not a predictor of hernia development.

Most of the risk factors identified related specifically to incisional hernia post stoma reversal are uncorrectable. It is therefore imperative that surgical techniques are evaluated to ensure we can optimise each patients risk.

\section{SUTURE TECHNIQUES TO PREVENT INCISIONAL HERNIA}

The strength of any sutured wound increases with a higher suture length to wound length (SL/WL) ratio, and is calculated as below:

(Original length of suture (Length of suture remnants at the starting knot + Length of suture remnants at the finishing knot)) / Length of skin incision

Wound dehiscence is unlikely to occur when there is a higher SL/WL ratio (34). Any stomal closure wound should therefore be closed with a SL/WL ratio of greater than 4 in order to prevent incisional hernia formation.

Continuous and interrupted are two suture methods commonly used for abdominal wall closure. Continuous suture method can be advantageous as it uses less suture material and is quicker to perform, but the strength of the entire closure is reliant on the one knot being secure. A meta-analysis published in 2010 included 14 randomised controlled trials with 7,711 patients examined continuous versus interrupted suture method in elective midline laparotomy closure (16). A reduced incisional hernia rate was seen in those with a continuous suture closure technique at a minimum of 12 month follow-up (OR 0.59; 95\% CI 0.43-0.82). The authors noted however, that many of the studies that were included were at high risk of bias due to the use of rapidly absorbable suture material in the interrupted suture intervention arm.

Suture bite size has been evaluated in the setting of midline closure in the STITCH Trial (43). Five-hundred and forty-five patients undergoing midline incisional surgery were randomised to either small bite suture placement $(0.5 \mathrm{~cm}$ from the wound edge every $0.5 \mathrm{~cm}$ along the wound, taking aponeurosis within the bite) or large bite suture placement $(1 \mathrm{~cm}$ from wound edge every $1 \mathrm{~cm}$ along the wound). There was lower incisional hernia rate at 1 year follow-up in the small bite group compared the large bite group ( 13 vs $21 \%$, OR $0.52 ; 95 \%$ CI $0.31-0.87$ ). The small bite technique was also found to have a higher SL/WL (5.0 vs $4.3 ; p<0.0001)$. However, the study was quasi randomised (alternated per calendar week) and only included 1 year follow-up. It should also be noted that differing suture material was used in both groups and that in the smaller bite group, aponeurosis only was closed in the bites, therefore posing further questions as to whether the bite size was responsible for the difference in the incisional hernia rate or whether suture material or layers included in the bite played a role.

Other suturing methods have been proposed for midline closure. The Hughes Abdominal Repair Trial (HART) randomised controlled trial, is comparing a far-near-near-far interrupted suture used alongside a continuous suture compared to continuous suture method alone in patients undergoing midline incisions $(44,45)$. The trial is due to report on its primary outcome of clinically detected incisional hernia at 12 month follow-up in 2019.

To date there has been little evidence which has focused on suture method in stoma site closure. Efforts should be made to investigate the various suture techniques described above within the setting of stoma closures.

\section{CAN PROPHYLACTIC MESH PLACEMENT REDUCE THE RISK?}

There are a wide variety of synthetic meshes that are commercially available for use in hernia repairs, these include non-absorbable (either light weight/large pore size or heavy weight/small pore size), absorbable or biological. Meshes can be placed in several locations, either onlay, retromuscular or preperitoneal. There is also a variety of mesh fixation techniques including, suture fixation (both interrupted or continuous technique and suture material choice), tacking staples, fibrin glue, no fixation or self-fixation meshes. There is a minimal evidence base that adequately examines the use of mesh (or the technique for its placement) for prevention of incisional hernia formation post ostomy reversal, which is discussed below.

A retrospective cohort case control study investigated whether the placement of an onlay prophylactic polypropylene mesh at time of stoma closure reduced the rate of incisional hernia after ileostomy closure (38). The study included 83 cases of ileostomy closure over a 5 year period (47 patients with mesh reinforcement and 36 without) with a median follow-up of 18.2 months (IQR 11.7-30.8 months). The rate of incisional hernia was $6.4 \%$ in the 
mesh group compared with $36.1 \%$ in the control group, as detected by combined clinical examination and CT imaging (OR 8.29, 95\% CI 2.14-32.08; $p=0.001$ ).

There have been a number of concerns raised with the use of prophylactic mesh placement and infection due to wound contamination with enterocutaneous flora. It has been proposed that a biological, collagen-based mesh may be a safer adjunct for use in stoma site closures, as the tissue matrix should become incorporated into the host tissue (46). A blinded, case-matched study assessed the retromuscular placement of bioprosthetic collagen porcine mesh at loop ileostomy reversal (47). Thirty patients received mesh reinforcement, and were compared to 64 control patients who received standard stoma closure without mesh placement. At twelvemonth follow-up, the incidence of incisional hernia on both clinical examination and CT imaging was reduced in mesh group, but was not statistically significant ( 3 vs $16 \%(p=0.17)$ and 3 vs $19 \%(p=0.43)$ respectively). The ROCSS (Reinforcement of Closure of Stoma Site) trial is a randomised controlled trial within the UK assessing the use of intra-abdominal biological mesh reinforcement in comparison to standard closure of stoma sites (48). Recruitment is complete, with 790 patients randomised, and the trial should be reporting its primary outcome of clinical herniation at 2 year follow-up in 2018.

A feasibility study from The Netherlands has also considered whether mesh placement at the time of temporary stoma formation can prevent incisional hernias following stoma closure (49). Ten patients who underwent low anterior resection received an intraperitoneal composite parastomal mesh reinforcement at time of defunctioning stoma formation. At median follow-up of 26 months (14-29) after stoma reversal, no incisional hernias were detected either clinically or on ultrasound imaging. However, it should be noted that adhesions to the mesh were present in all patients in the study and covered a median of $25 \%$ of the mesh surface. Whilst the authors commented that no adhesion related morbidity occurred, one patient required a laparotomy in order to mobilise the bowel at time of reversal due to adhesions at the curled up mesh border. Further studies are certainly required to investigate whether mesh placement at time of stoma formation may be of benefit to prevent incisional hernia following reversal, with larger sample size to assess morbidity related to mesh location. Extraperitoneal placement should also be considered in this setting as it may reduce any potential for mesh-related adhesion morbidity.

It should be noted that in situations of planned elective formation of a defunctioning loop ileostomy, then there is a necessity for early function and no stenosis and we also believe that at the time of subsequent closure that a small hernia facilitates the mobilisation.

\section{PREVENTION OF SURGICAL-SITE INFECTION WITH SKIN CLOSURE METHODS}

Whilst the presence of SSI has not been proven to be a risk factor specifically for the development of an incisional hernia at the site of stoma closure, it is accepted as such in incisional hernias in general $(33,34)$. Methods to prevent SSI should therefore be welcomed within stoma reversal surgeries, particularly as there is pre-existing contamination of the wound from the open bowel lumen. Secondary intention skin healing, several primary closure methods ("air-tight" primary closure, "loose" primary closure or "delayed" primary closure), or a hybrid method utilising a purse-string suture (also known as "circular" closure) have been suggested as methods to deal with the skin at the stoma site following reversal (50-52).

Three meta-analysis have compared circular closure with primary skin closure following stoma reversal. McCarten et al (2013) included 2 randomised controlled trials and 4 case controlled series with a total of 403 patients (53). Circular closure suture technique resulted in an $80 \%$ reduction in the rate of SSI when compared to primary closure, 2.4 versus $29.6 \%$, respectively (OR 0.083 , 95\% CI $0.03-0.21 ; p<0.001)$. Patients who had circular closure also reported a greater satisfaction with cosmetic outcome (standard mean difference $=0.47$ on a patient self-reported assessment of cosmetic outcome using a ten-point visual analogue scale, 95\% CI $0.15-0.79 ; p=0.005)$. Sajid et al (2014) included 3 randomised controlled trials comparing circular closure versus primary skin closure in ileostomy reversal wounds (54). SSI was found to be lower in circular closure (101 patients) when compared to primary closure (105 patients) (OR 0.10, 95\% CI 0.03-0.33; $p<0.0001$ ). Hsieh et al (2015) performed a meta-analysis of 4 randomised controlled trials with 319 participants (55). Circular closure had a lower incidence of SSI when compared to primary closure (Risk difference $0.25,95 \%$ CI -0.36 to $-0.15 ; p<0.00001$ ) and higher satisfaction with cosmetic outcomes (standard mean difference 0.7 , $95 \%$ CI $0.13-1.27, p=0.02$ ). The "STOMA" trial, a RCT published in 2017, also found a similar result with higher rates of SSI at 30 days in the primary closure arm compared to circular closure $(8 / 27(30 \%)$ versus $3 / 34(8 \%) ; p=0.03)(56)$.

A further systematic review and meta-analysis included original articles ( 3 randomised controlled trials and 12 retrospective reviews) comparing multiple skin closure methods, including primary closure, primary closure with drain, secondary closure, delayed primary closure, loose primary closure and circular closure with a total of 2,921 patients (57). In multiple treatments meta-analysis (random effects) and sensitivity analysis primary closure and delayed primary closure were ranked the worse choices for skin closure, with circular closure ranked the best choice for skin closure.

Circular closure has been hypothesised to decrease rate of SSI as it provides a route for drainage of wound contaminants whilst also providing a higher degree of wound apposition when compared to secondary intention healing. It should also be noted the primary outcome in all the included studies in the meta-analyses was SSI and not incisional hernia rate and many of the studies included were found to be of moderate to high risk of bias. Further studies should focus on well-conducted randomised controlled trial with outcomes measure of stoma site herniation.

\section{DOES MECHANICAL BOWEL PREPARATION PLAY A ROLE WITH SURGICAL-SITE INFECTIONS?}

A mulitcentre retrospective study of 272 children who underwent colostomy takedown evaluated the role of mechanical 
bowel preparation in SSI rates (58). Polyethylene glycol bowel preparation was administered to 187 children pre-operatively and 85 children were classified as controls as they did not receive mechanical bowel preparation. All patients received intravenous antibiotics. Fifty two percent of those who had mechanical bowel preparation received oral antibiotics, whereas no patients in the control group had oral antibiotics. The incidence of SSI was found to be significantly higher in the mechanical bowel preparation group when compared to the controls (14.4 versus $5.8 \% ; p=0.04)$.

Shah et al (2016) performed a prospective randomised study on mechanical bowel preparation within children undergoing elective bowel resection, including stoma closure (59). Eighteen patients were randomised to receive mechanical bowel preparation and 14 were randomised to receive none. The rate of SSI was $11.1 \%$ in the treatment arm compared with $21.4 \%$ in the group arm, however, this was not found to be statistically significant $(p=0.63)$. The results did not allow extraction of data specific to the cohort of those who underwent stoma closure only and it should be recognized that the sample size was very small and therefore the results of this randomised controlled trial should be taken with caution. Both of these studies were within the paediatric population, which most often have different underlying pathology responsible for the need for stoma formation and therefore the results may not be applicable in adults. There is evidence to support that the use of preoperative mechanical bowel preparation combined with oral antibiotics reduces surgical site infection after colorectal resectional surgery, however this has not been evaluated

\section{REFERENCES}

1. Chow A, Tilney HS, Paraskeva P, Jeyarajah S, Zacharakis E, Purkayastha S. The morbidity surrounding reversal of defunctioning ileostomies: a systematic review of 48 studies including 6,107 cases. Int J Colorectal Dis (2009) 24(6):71123. doi: $10.1007 / \mathrm{s} 00384-009-0660-\mathrm{z}$

2. Tilney HS, Sains PS, Lovegrove RE, Reese GE, Heriot AG, Tekkis PP. Comparison of outcomes following ileostomy versus colostomy for defunctioning colorectal anastomoses. World J Surg (2007) 31(5):1143-52. doi: 10.1007/s00268-006-0218-y

3. Rondelli F, Reboldi P, Rulli A, Barberini F, Guerrisi A, Izzo L, et al. Loop ileostomy versus loop colostomy for fecal diversion after colorectal or coloanal anastomosis: a meta-analysis. Int J Colorectal Dis (2009) 24(5):479-88. doi: 10.1007/s00384-009-0662-x

4. Guenaga KF, Lustosa SA, Saad SS. Ileostomy or colostomy for temporary decompression of colorectal anastomosis. Cochrane Database Syst Rev (2007) 1:CD004647.

5. Muysoms FE, Miserez M, Berrevoet F, Campanelli G, Champault GG, Chelala $\mathrm{E}$, et al. Classification of primary and incisional abdominal wall hernias. Hernia (2009) 13(4):407-14. doi: 10.1007/s10029-009-0518-x

6. van Ramshorst GH, Eker HH, Hop WC, Jeekel J, Lange JF. Impact of incisional hernia on health-related quality of life and body image: a prospective cohort study. Am J Surg (2012) 204(2):144-50. doi: 10.1016/j.amjsurg.2012.01.012

7. van 't Riet M, Steyerberg EW, Nellensteyn J, Bonjer HJ, Jeekel J. Meta-analysis of techniques for closure of midline abdominal incisions. Br J Surg (2002) 89(11):1350-6. doi: 10.1046/j.1365-2168.2002.02258.x

8. Burger JW, Luijendijk RW, Hop WC, Halm JA, Verdaasdonk EG, Jeekel J. Long-term follow-up of a randomized controlled trial of suture versus mesh repair of incisional hernia. Ann Surg (2004) 240(4):176-83. doi: 10.1097/01.sla. 0000141193.08524.e7 within patients for ostomy reversal $(60,61)$. Well conducted studies evaluating the use of mechanical bowel preparation, with or without the combination of oral antibiotics for stoma reversal should be undertaken.

\section{CONCLUSIONS}

Incisional hernias following ostomy reversal occur frequently. Despite this, the evidence base on the subject is limited. Many recognised risk factors for their development are related to patient factors and are not easily correctable. There is a limited amount of evidence to suggest that prophylactic mesh reinforcement may be of benefit to reduce the post stoma closure incisional hernia rate but a further large scale randomised controlled trial is due to report in the near future. There appears to be weak evidence to suggest that surgeons should favour circular, or "purse-string" closure of the skin following stoma closure in order to reduce the risk of SSI, which in turn may reduce incisional hernia formation. There remains the need for further evidence in relation to suture technique, skin closure techniques, mechanical bowel preparation and oral antibiotic prescription focusing on incisional hernia development as an outcome measure.

\section{AUTHOR CONTRIBUTIONS}

\author{
$\mathrm{RH}$ and JT wrote the review
}

9. NHS Digital, Hospital Episode Statistics for England. Admitted Patient Care Statistics, 2015-16. (2016).

10. Finan KR, Kilgore ML, Hawn MT. Open suture versus mesh repair of primary incisional hernias: a cost-utility analysis. Hernia (2009) 13(2):173-82. doi: 10.1007/s10029-008-0462-1

11. Gillion JF, Sanders D, Miserez M, Muysoms F. The economic burden of incisional ventral hernia repair: a multicentric cost analysis. Hernia (2016) 20(6):819-30. doi: 10.1007/s10029-016-1480-Z

12. Bhangu A, Nepogodiev D,Futaba K, West Midlands Research Collaborative. Systematic review and meta-analysis of the incidence of incisional hernia at the site of stoma closure. World J Surg (2012) 36(5):973-83. doi: 10.1007/s00268-0121474-7

13. Nguyen MT, Phatak UR, Li LT, Hicks SC, Moffett JM, Arita NA, et al. Review of stoma site and midline incisional hernias after stoma reversal. J Surg Res (2014) 190(2):504-9. doi: 10.1016/j.jss.2014.01.046

14. Bhangu A, Fletcher L, Kingdon S, Smith E, Nepogodiev D, Janjua U. A clinical and radiological assessment of incisional hernias following closure of temporary stomas. Surgeon (2012) 10(6):321-5. doi: 10.1016/j. surge.2012.01.001

15. Cingi A, Cakir T, Sever A, Aktan AO. Enterostomy site hernias: a clinical and computerized tomographic evaluation. Dis Colon Rectum (2006) 49(10):155963. doi: 10.1007/s10350-006-0681-4

16. Diener MK, Voss S, Jensen K, Büchler MW, Seiler CM. Elective midline laparotomy closure: the INLINE systematic review and meta-analysis. Ann Surg (2010) 251(5):843-56. doi: 10.1097/SLA.0b013e3181d973e4

17. Höer J, Lawong G, Klinge U, Schumpelick V. [Factors influencing the development of incisional hernia. A retrospective study of 2,983 laparotomy patients over a period of 10 years]. Chirurg (2002) 73(5):474-80. doi: 10.1007/ s00104-002-0425-5 
18. Armstrong CP, Dixon JM, Duffy SW, Elton RA, Davies GC. Wound healing in obstructive jaundice. Br J Surg (1984) 71(4):267-70. doi: 10.1002/ bjs. 1800710405

19. Bayer I, Ellis H. Jaundice and wound healing: an experimental study. Br J Surg (1976) 63(5):392-6. doi: 10.1002/bjs.1800630515

20. Sørensen LT, Hemmingsen UB, Kirkeby LT, Kallehave F, Jørgensen LN. Smoking is a risk factor for incisional hernia. Arch Surg (2005) 140(2):119-23. doi: 10.1001/archsurg.140.2.119

21. Rosch R, Junge K, Knops M, Lynen P, Klinge U, Schumpelick V. Analysis of collagen-interacting proteins in patients with incisional hernias. Langenbecks Arch Surg (2003) 387(11-12):427-32. doi: 10.1007/s00423-0020345-3

22. Israelsson LA, Jonsson T. Overweight and healing of midline incisions: the importance of suture technique. Eur J Surg (1997) 163(3):175-80.

23. Franchi M, Ghezzi F, Buttarelli M, Tateo S, Balestreri D, Bolis P. Incisional hernia in gynecologic oncology patients: a 10-year study. Obstet Gynecol (2001) 97(5 Pt 1):696-700.

24. Mäkelä JT, Kiviniemi H, Juvonen T, Laitinen S. Factors influencing wound dehiscence after midline laparotomy. Am J Surg (1995) 170(4):387-90. doi: 10.1016/S0002-9610(99)80309-2

25. Junge K, Klinge U, Klosterhalfen B, Rosch R, Stumpf M, Schumpelick V. Review of wound healing with reference to an unrepairable abdominal hernia. Eur J Surg (2002) 168(2):67-73. doi: 10.1080/11024150252884269

26. Niggebrugge AH, Trimbos JB, Hermans J, Steup WH, van de Velde CJ. Influence of abdominal-wound closure technique on complications after surgery: a randomised study. Lancet (1999) 353(9164):1563-7. doi: 10.1016/ S0140-6736(98)10181-2

27. Daly JW. Dehiscence, evisceration, and other complications. Clin Obstet Gynecol (1988) 31(3):754-60. doi: 10.1097/00003081-198809000-00023

28. Adell-Carceller R, Segarra-Soria MA, Pellicer-Castell V, Marcote-Valdivieso E, Gamón-Giner R, Martín-Franco MA, et al. [Incisional hernia in colorectal cancer surgery. Associated risk factors]. Cir Esp (2006) 79(1):42-5.

29. Bucknall TE, Cox PJ, Ellis H. Burst abdomen and incisional hernia: a prospective study of 1129 major laparotomies. Br Med J (1982) 284(6320):9313. doi: $10.1136 / \mathrm{bmj} .284 .6320 .931$

30. Takagi H, Sugimoto M, Kato T, Matsuno Y, Umemoto T. Postoperative incision hernia in patients with abdominal aortic aneurysm and aortoiliac occlusive disease: a systematic review. Eur J Vasc Endovasc Surg (2007) 33(2):177-81. doi: 10.1016/j.ejvs.2006.07.009

31. Adye B, Luna G. Incidence of abdominal wall hernia in aortic surgery. Am J Surg (1998) 175(5):400-2.

32. Lamont PM, Ellis $\mathrm{H}$. Incisional hernia in re-opened abdominal incisions: an overlooked risk factor. Br J Surg (1988) 75(4):374-6. doi: 10.1002/ bjs. 1800750426

33. Carlson MA. Acute wound failure. Surg Clin North Am (1997) 77(3):607-36. doi: 10.1016/S0039-6109(05)70571-5

34. Israelsson LA, Jonsson T. Suture length to wound length ratio and healing of midline laparotomy incisions. Br J Surg (1993) 80(10):1284-6. doi: 10.1002/ bjs. 1800801020

35. Cingi A, Solmaz A, Attaallah W, Aslan A, Aktan AO. Enterostomy closure site hernias: a clinical and ultrasonographic evaluation. Hernia (2008) 12(4):401-5. doi: 10.1007/s10029-008-0355-3

36. Guzmán-Valdivia G. Incisional hernia at the site of a stoma. Hernia (2008) 12(5):471-4. doi: 10.1007/s10029-008-0378-9

37. Schreinemacher MH, Vijgen GH, Dagnelie PC, Bloemen JG, Huizinga BF, Bouvy ND. Incisional hernias in temporary stoma wounds: a cohort study. Arch Surg (2011) 146(1):94-9. doi: 10.1001/archsurg.2010.281

38. Liu DS, Banham E, Yellapu S. Prophylactic mesh reinforcement reduces stomal site incisional hernia after ileostomy closure. World J Surg (2013) 37(9):2039_ 45. doi: 10.1007/s00268-013-2109-3

39. Liang MK, Li LT, Avellaneda A, Moffett JM, Hicks SC, Awad SS. Outcomes and predictors of incisional surgical site infection in stoma reversal. JAMA Surg (2013) 148(2):183-9. doi: 10.1001/jamasurgery.2013.411

40. Sharp SP, Francis JK, Valerian BT, Canete JJ, Chismark AD, Lee EC. Incidence of ostomy site incisional hernias after stoma closure. Am Surg (2015) 81(12):1244-8.

41. Brook AJ, Mansfield SD, Daniels IR, Smart NJ. Incisional hernia following closure of loop ileostomy: The main predictor is the patient, not the surgeon. Surgeon (2018) 16(1):20-6. doi: 10.1016/j.surge.2016.03.004
42. Huang C, Ogawa R. The link between hypertension and pathological scarring: does hypertension cause or promote keloid and hypertrophic scar pathogenesis? Wound Repair Regen (2014) 22(4):462-6. doi: 10.1111/ wrr. 12197

43. Deerenberg EB, Harlaar JJ, Steyerberg EW, Lont HE, van Doorn HC, Heisterkamp J, et al. Small bites versus large bites for closure of abdominal midline incisions (STITCH): a double-blind, multicentre, randomised controlled trial. Lancet (2015) 386(10000):1254-60. doi: 10.1016/S01406736(15)60459-7

44. Cornish J, Harries RL, Bosanquet D, Rees B, Ansell J, Frewer N, et al. Hughes Abdominal Repair Trial (HART) - Abdominal wall closure techniques to reduce the incidence of incisional hernias: study protocol for a randomised controlled trial. Trials (2016) 17(1):454. doi: 10.1186/s13063-016-1573-0

45. Harries RL, Cornish J, Bosanquet D, Rees B, Horwood J, Islam S, et al. Hughes Abdominal Repair Trial (HART)-abdominal wall closure techniques to reduce the incidence of incisional hernias: feasibility trial for a multicentre, pragmatic, randomised controlled trial. BMJ Open (2017) 7(12):e017235. doi: 10.1136/ bmjopen-2017-017235

46. Bhangu A, Futaba K, Patel A, Pinkney T, Morton D. Reinforcement of closure of stoma site using a biological mesh. Tech Coloproctol (2014) 18(3):305-8. doi: 10.1007/s10151-013-1001-3

47. Maggiori L, Moszkowicz D, Zappa M, Mongin C, Panis Y. Bioprosthetic mesh reinforcement during temporary stoma closure decreases the rate of incisional hernia: a blinded, case-matched study in 94 patients with rectal cancer. Surgery (2015) 158(6):1651-7. doi: 10.1016/j.surg.2015.07.004

48. Reinforcement of Closure of Stoma Site (ROCSS) Collaborative and the West Midlands Research Collaborative. Feasibility study from a randomized controlled trial of standard closure of a stoma site vs biological mesh reinforcement Colorectal Dis (2016) 18(9):889-96. doi: 10.1111/codi.13310

49. van Barneveld KW, Vogels RR, Beets GL, Breukink SO, Greve JW, Bouvy ND, et al. Prophylactic intraperitoneal mesh placement to prevent incisional hernia after stoma reversal: a feasibility study. Surg Endosc (2014) 28(5):1522-7. doi 10.1007/s00464-013-3346-0

50. Hackam DJ, Rotstein OD. Stoma closure and wound infection: an evaluation of risk factors. Can J Surg (1995) 38(2):144-8.

51. Vermulst N, Vermeulen J, Hazebroek EJ, Coene PP, van der Harst E. Primary closure of the skin after stoma closure. Management of wound infections is easy without (long-term) complications. Dig Surg (2006) 23(4):255-8. doi: $10.1159 / 000095399$

52. Banerjee A. Pursestring skin closure after stoma reversal. Dis. Colon Rectum (1997) 40(8):993-4. doi: 10.1007/BF02051210

53. Mccartan DP, Burke JP, Walsh SR, Coffey JC. Purse-string approximation is superior to primary skin closure following stoma reversal: a systematic review and meta-analysis. Tech Coloproctol (2013) 17(4):345-51. doi: 10.1007/s10151012-0970-y

54. Sajid MS, Bhatti MI, Miles WF. Systematic review and meta-analysis of published randomized controlled trials comparing purse-string vs conventional linear closure of the wound following ileostomy (stoma) closure. Gastroenterol Rep (2015) 3(2):156e61. doi: 10.1093/gastro/gou038

55. Hsieh MC, Kuo LT, Chi CC, Huang WS, Chin CC. Pursestring closure versus conventional primary closure following stoma reversal to reduce surgical site infection rate: a meta-analysis of randomized controlled trials. Dis Colon Rectum (2015) 58(8):808-15. doi: 10.1097/DCR.0000000000000401

56. O'Leary DP, Carter M, Wijewardene D, Burton M, Waldron D, Condon E, et al. The effect of purse-string approximation versus linear approximation of ileostomy reversal wounds on morbidity rates and patient satisfaction: the 'STOMA' trial. Tech Coloproctol (2017) 21(11):863-8. doi: 10.1007/s10151017-1713-x

57. Li LT, Hicks SC, Davila JA, Kao LS, Berger RL, Arita NA, et al. Circular closure is associated with the lowest rate of surgical site infection following stoma reversal: a systematic review and multiple treatment meta-analysis. Colorectal Dis (2014) 16(6):406e16. doi: 10.1111/codi.12556

58. Serrurier K, Liu J, Breckler F, Khozeimeh N, Billmire D, Gingalewski C, et al. A multicenter evaluation of the role of mechanical bowel preparation in pediatric colostomy takedown. J Pediatr Surg (2012) 47(1):190-3. doi: 10.1016/j. jpedsurg.2011.10.044

59. Shah M, Ellis CT, Phillips MR, Marzinsky A, Adamson W, Weiner T, et al. Preoperative bowel preparation before elective bowel resection or ostomy closure 
in the pediatric patient population has no impact on outcomes: a prospective randomized study. Am Surg (2016) 82(9):801-6.

60. Kiran RP, Murray AC, Chiuzan C, Estrada D, Forde K. Combined preoperative mechanical bowel preparation with oral antibiotics significantly reduces surgical site infection, anastomotic leak, and ileus after colorectal surgery. Ann Surg (2015) 262(3):416-25. doi: 10.1097/SLA.0000000000001416

61. Vo E, Massarweh NN, Chai CY, Tran Cao HS, Zamani N, Abraham S, et al. Association of the addition of oral antibiotics to mechanical bowel preparation for left colon and rectal cancer resections with reduction of surgical site infections. JAMA Surg (2018) 153(2):114. doi: 10.1001/jamasurg.2017.3827
Conflict of Interest Statement: The authors declare that the research was conducted in the absence of any commercial or financial relationships that could be construed as a potential conflict of interest.

Copyright $\odot 2018$ Harries and Torkington. This is an open-access article distributed under the terms of the Creative Commons Attribution License (CC BY). The use, distribution or reproduction in other forums is permitted, provided the original author $(s)$ and the copyright owner are credited and that the original publication in this journal is cited, in accordance with accepted academic practice. No use, distribution or reproduction is permitted which does not comply with these terms. 